\title{
Modelado de Procesos de Negocio para la Gestión de Patentes en Universidades
}

\author{
Rodolfo Schmal ${ }^{(1)}$, Socorro López ${ }^{(2)}$, Fernando Cabrales ${ }^{(3)}$ y Diego Acuña ${ }^{(4)}$ \\ (1) Facultad Ciencias Empresariales, U. de Talca, Talca-Chile (email: rschmal@utalca.cl) \\ (2) Facultad Ciencias Económicas, U. de Antioquia, Medellín-Colombia (email: socolopez@yahoo.es) \\ (3) Corporación de Fomento de la Producción, CORFO, Arica-Chile (email: fcabrale@uta.cl) \\ (4) Escuela Ing. Informática Empresarial, U. de Talca, Talca-Chile \\ (email: dacuna@alumnos.utalca.cl)
}

Recibido Nov. 17, 2009; Aceptado Ene. 15, 2010; Versión Final recibida Abr. 16, 2010

\begin{abstract}
Resumen
El objetivo del trabajo presentado fue elaborar un modelo de procesos de negocios para la gestión de las patentes resultantes de las ideas y proyectos que surgen en las universidades. Para abordar el diseño de este modelo, se realizaron entrevistas, encuestas y visitas a sitios Web de diversas universidades con el fin de determinar el conjunto de actividades desde que surge una idea susceptible de ser patentada hasta que la patente es explotada en el mercado. Esta identificación de actividades y su análisis fue efectuada mediante el modelo de procesos por regulación, el que posteriormente fue traducido a un modelo en BPMN (Business Process Modeling Notation). Se muestra que el modelo propuesto facilita la gestión de las patentes en las universidades.
\end{abstract}

Palabras clave: procesos de negocios, derechos de propiedad intelectual, gestión de patentes, modelos de procesos

\section{Modeling of Business Processes for the Management of Patents in Universities}

\begin{abstract}
The objective of this paper was the proposal of a business process model for the management of patents resulting from ideas or projects developed in universities. For the design of the model, several actions were developed: surveys, interviews, and analysis of web sites, with the aim of determining the set of activities followed in other institutions from the original idea until patent exploitation. This information and its analysis were done using the process modelling by regulation, later translated into a model in BPMN (Business Process Modeling Notation). It is shown that the proposed model facilitates the management of patents in universities.
\end{abstract}

Keywords: business process, intellectual property rights, patents management, process models 


\section{INTRODUCCIÓN}

La Ley Bayh Dole (B.D.A. por sus siglas en inglés), y el surgimiento de nuevos sectores tecnológicos altamente vinculados con la ciencia (bios y nanos), han tenido considerables efectos en el comportamiento de las universidades respecto del uso de las patentes como mecanismo de difusión y transferencia tecnológica de sus resultados de investigación, lo cual a su vez ha generado un intenso debate entre académicos, tanto a favor como en contra de la privatización del conocimiento y también sobre los asuntos éticos de las patentes (Calderini et al., 2009). Este debate y el incremento de las patentes universitarias, ha dado origen a múltiples estudios, desde diferentes perspectivas y propósitos (López, 2008).

La Ley Bayh Dole impactó la investigación que se desarrolló en las universidades, elevando la importancia de la investigación aplicada en sus laboratorios e impulsando la comercialización de los resultados de la investigación. Diversos estudios, destacándose los de Henderson et al., (1998), Sampat et al. (2003), Jensen y Thursby (2001), Nelson (2004), Mowery et al. (2001), Sieguel et al. (2004), han tratado de evaluar el impacto de la ley Bayh Dole en la investigación y más concretamente en la patentabilidad universitaria, observándose que con posterioridad a la ley se generalizó la creación de Oficinas de Transferencia Tecnológica en las Universidades estadounidenses, se incrementó significativamente la cantidad de patentes concedidas, y lo que es más importante, el volumen y valor de los contratos establecidos con las empresas (Siegel et al., 2003).

Baldini (2006), realiza una amplia revisión (125 artículos entre 1980 y 2004) de la literatura sobre patentes y licencias universitarias, y respecto a la gestión de patentes reconoce los estudios de Argyres y Liebeskind (1998); Branscomb et al. (1999); Mowery y Sampat (2001); Mowery et al. (2004). Entre sus hallazgos más importantes se destaca que las universidades requieren de un contexto regional y nacional fértil para desarrollar sus actividades de patentes y licencias; y que el éxito de los Estados Unidos en materia de patentes universitarias, no puede ser imitado o impulsado sólo con la aplicación aislada de algunos de los incentivos tales como la transferencia de los derechos de propiedad intelectual de los inventores a las instituciones (o viceversa).

Azagra y Romero (2009), clasifican los diferentes estudios sobre patentes universitarias desde la perspectiva económica e histórica, para analizar luego los determinantes institucionales de las patentes en el principal Organismo Público de Investigación de España. Allí señalan que normalmente estos estudios históricos se han ocupado de patentes generadas en el entorno universitario, con el objeto de explicar la incidencia de la investigación académica en la transferencia de tecnología, mientras que la mayor parte de los análisis económicos han buscado determinar la influencia de diferentes factores sobre la generación de patentes en el sistema público de investigación y desarrollo (I+D), algunas veces tratando de cuantificarla mediante estimaciones econométricas (Foltz et al., 2000; Carlsson et al., 2002; Payne y Siow, 2003; Coupé, 2003; Baldini et al., 2004; Azagra y Romero, 2009).

En la revisión de la literatura realizada, se encontraron algunos elementos en el campo de la gestión, tales como la problemática sobre incentivos (Jensen y Thursby, 2001; Azagra et al., 2001; OCDE, 2003); las patentes como instrumentos en las relaciones Universidad - Empresa (Azagra, 2004; Tijssen, 2004; Cohen et al., 2002) y la relación entre los niveles de patentabilidad con la importancia otorgada a organismos de enlace como son las oficinas de transferencia tecnológica (OTT) (Siegel et al., 2003) y propuestas de instrumentos para la gestión de los derechos de propiedad intelectual en los organismos públicos de investigación (Fernández y Represa, 1998).

Así mismo, Iversen et al. (2007), Gulbrandsen et al. (2005), Cohen y Walsh (2001), Bessen y Hunt (2004), en sus trabajos buscaron específicamente conocer los comportamientos y las motivaciones de los sectores tecnológicos y de los académicos en sus decisiones de patentar. Las motivaciones de las universidades hacia el patentamiento tienen otro trasfondo, pues a pesar que los universitarios tengan cierta aversión a patentar, Geuna y Nesta (2006) aseguran que los beneficios de patentar superan a los costes. En un estudio empírico realizado por López et al. (2006), según los gestores de las oficinas de gestión de la investigación y de patentes, entre las razones que las universidades 
tienen para patentar, en orden de importancia fueron: el prestigio e impacto que genera para la universidad, la orientación que tienen hacia la investigación aplicada, y los beneficios que se esperan de la producción de patentes.

En el caso contrario, entre las desventajas al patentar se destacó como principal, la burocracia para operar el sistema, los altos costes de investigación, registro y mantenimiento, y el tiempo que se requiere para el proceso. Los investigadores, así como los gestores de la I+D de las universidades, creen que la universidad no patenta principalmente por inexperiencia en materia de desarrollo de productos patentables, pero también incide la ausencia de empresas innovadoras en el área de influencia de la universidad; cuando la investigación que se realiza está dirigida fundamentalmente a la ciencia básica; y también tiene que ver con la baja patentabilidad la posición filosófica frente al papel que debe jugar la Universidad en la creación y difusión del conocimiento así como la existencia de un sistema de patentes burocrático, poco fiable y una escasa claridad en torno a los incentivos para los inventores.

En resumen, la tendencia por incrementar los niveles de patentes en las universidades ha centrado el debate sobre la conveniencia o no de patentar, hacia el rol de las universidades, las cuales sin abandonar su misión inicial, continúan como responsables de la creación, transmisión y difusión del conocimiento, pero además en un contexto de comercialización del conocimiento las universidades están comprometidas con el impulso, la ejecución y apoyo al desarrollo tecnológico de las empresas de sus propios países. Por ello los estudios sobre las patentes universitarias en muchos casos se han enfocado por un lado, en su análisis como indicador de innovación y la explicación de la incidencia de la investigación académica en la transferencia de tecnología, pero por otro lado, los estudios también pretenden contribuir en los aspectos normativos destinados a mejorar la gestión de patentes universitarias.

Las universidades latinoamericanas, aunque no tienen tradición de patentar, no pueden eximirse de evaluar en profundidad este cambio de contexto, particularmente cuando los tratados de libre comercio (TLC) que se están suscribiendo con Estados Unidos, les obliga a ajustar sus marcos jurídicos a los convenios internacionales en materia de Derechos de Propiedad Intelectual y específicamente en el campo de las patentes.

De hecho, la universalización de la legislación sobre propiedad intelectual puede verse, además de una imposición de los tiempos, como una oportunidad para los países en desarrollo con miras a participar de un negocio de grandes perspectivas.

En este contexto las universidades latinoamericanas tienen que dimensionar su responsabilidad en el desarrollo técnico de los países con el apoyo al Estado en la construcción de políticas y estrategias para la aplicación de los derechos de propiedad intelectual. Si bien la patentabilidad no es necesariamente el camino más mediato, las universidades deben prepararse para una adecuada gestión de las patentes en el marco de la explotación de los resultados de la investigación.

Bajo este marco, en el año 2008 la Universidad de Talca (Chile), ha creado la Dirección de Transferencia y Centros Tecnológicos, la que tiene como una de sus responsabilidades la gestión de los derechos de propiedad intelectual de los resultados de los proyectos de investigación que desarrollan sus académicos. Por ello esta unidad está interesada en implementar un modelo que contribuya a la creación de una institucionalidad dotada de capacidades de gestión para la transferencia tecnológica desde las universidades, enmarcada en la ampliación y profundización de vías de relacionamiento con los agentes de innovación.

\section{EL MODELO}

Una revisión de la literatura en torno a la gestión de las patentes en las universidades que registran mayores niveles de patentabilidad, entre las que destacan las estadounidenses, se observa que los trabajos desarrollados están más centrados en las estructuras de las unidades organizacionales y de las formas de vinculación con las empresas, que en la gestión de los procesos implicados en la patentabilidad (Chang et al., 2006; Macho-Stadler et al., 2007; McAdam et al., 2005; Thursby et al., 
2009). Lo señalado se confirma como consecuencia de interpretar y debatir la información derivada de la aplicación de encuestas a 23 universidades (11 de Colombia, 5 de Chile y 7 de España); la revisión de páginas web universitarias; y de los estatutos de propiedad intelectual de la Universidad de Antioquia - Colombia, la Universidad Nacional de Colombia y la Universidad de Talca - Chile (Lopez et al., 2006).

En consecuencia, no se encontró, al menos en forma explícita, un modelo de gestión de los procesos de negocios implicados en la patentabilidad. Esto puede explicarse porque el modelamiento de procesos de negocio es una temática relativamente reciente al igual que el interés por la gestión de las patentes en las universidades. Sin embargo, a partir de las experiencias existentes en las universidades estadounidenses y europeas más involucradas con la patentabilidad y la vinculación con empresas (Tornatzky et al., 2002; Rasmussen et al., 2006), se observó la existencia de actividades comunes susceptibles de agruparse en procesos.

A pesar de que ya en 1960 se hizo mención a la importancia de los procesos de negocio (Levitt, 1960), recién en las últimas décadas ha cobrado relevancia en la gestión, puesto que su modelamiento posibilita que las organizaciones sean analizadas e integradas a través de sus procesos de negocios. Este enfoque ha dado origen a diversas metodologías de modelamiento de procesos, entre ellas la de análisis y diseño estructurado de sistemas (SSADM), los sistemas soft (SSM), los grafos con resultados y actividades interrelacionadas (GRAI) y simulaciones, las que hacen uso de diversas técnicas y herramientas tales como diagramas de flujo de datos (DFD), diagramas de rol de actividades (RAD), diagramas de interacción de roles (RID), cartas Gantt, definición integrada para el modelamiento funcional (IDEF), redes de Petri coloreadas (RPC), métodos orientados a objetos (MOO) y las técnicas de workflow, cuyas descripciones se pueden encontrar en Aguilar-Savén (2004).

Inicialmente el modelo fue diseñado haciendo uso del Modelamiento de Procesos por Regulación (MPR), el cual proporciona un modelo gráfico organizacional general basado en la teoría de sistemas y en patrones de procesos de regulación organizacional concordantes con experiencias y observaciones empíricas (Barros, 1995; López et al., 2009). Posteriormente se tradujo a BPMN (Business Process Modeling Notation) creada el año 2004 para proveer una notación de fácil comprensión a los usuarios -analistas de negocios que crean las primeras versiones de los procesos, los desarrolladores responsables de implementar la tecnología sobre la cual se ejecutarán estos procesos, y las personas que gestionarán y monitorearán dichos procesos- (White, 2008).

Davenport (1993) define un proceso como un "conjunto de actividades estructuradas y medibles destinadas a producir un resultado específico para un particular cliente o mercado". Por su parte, Hammer y Champy (1993) definen el concepto de Proceso de Negocio como un "conjunto de actividades que reciben uno o más insumos y crea un producto de valor para el cliente". Si bien la distinción entre actividad y proceso no es nítida, por lo general un proceso es visto como un conjunto de actividades o una macroactividad. Otra definición, entiende todo proceso como un "conjunto de tareas lógicamente relacionadas que existen para obtener un resultado bien definido dentro de un negocio" (Barros, 1995), en tanto que en ISO 9000 (2000) se define un proceso como un sistema de actividades subjetivamente identificados de manera tal que pueda gestionar holística y sistemáticamente el uso de recursos conducentes a transformar entradas en salidas.

Se asume que el propósito de todo sistema de gestión es el de regular el comportamiento de los recursos que manejan las organizaciones ante perturbaciones generadas por un entorno cambiante y no controlable. Estos recursos a regular (gestionar), son ingresados desde el entorno hacia la organización, para ser "operados" o "transformados" en su interior y devueltos al exterior. En el caso en estudio, el recurso que nos interesa regular es el conocimiento comercializable, susceptible de ser patentado.

Para identificar los procesos asociados a los derechos de propiedad intelectual se incurrió en los siguientes supuestos: 
a) El proceso por el cual una idea se transforma en un producto/proceso/servicio comercializado y explotado industrialmente en el mercado esta concebido bajo el modelo interactivo de innovación de Kline y Rosenberg (1986), pero para efectos del modelo presentaremos las actividades como un "continuum" que va desde la investigación básica, investigación aplicada, desarrollo, invento hasta su introducción al mercado. Se destaca que existen flujos e interrelaciones entre todas las actividades a través del proceso, en el que se identifican las siguientes etapas en los procesos de innovación tecnológica: desarrollo de la idea o concepto, factibilidad de prototipo, factibilidad de planta, y producción propiamente tal.

b) La responsabilidad de la etapa del desarrollo de la idea está centrada en los investigadores, preferentemente con la mirada puesta en un problema existente en el mundo real y no resuelto a la fecha. Este problema puede haber sido detectado por el propio investigador en su relación directa con la sociedad y las empresas, o bien, provenir de estas últimas desde la unidad responsable de la vinculación universidad-empresa-gobierno-sociedad.

c) En el interior de la universidad existe una instancia capaz de evaluar toda idea susceptible de dar origen a una patente.

d) Como resultado de la evaluación de una idea, su aprobación generará un conjunto de actividades conducentes a elevar una solicitud de patente ante las instancias correspondientes, en tanto que en caso de ser rechazada, se desecha.

e) Una vez que se considera que el proyecto es susceptible de generar una patente, se inicia el proceso de comercialización de la patente (venta o licencia) mediante la oferta a empresas o la formación de spin - off. Cabe señalar que la concesión de una patente no asegura la existencia de una innovación tecnológica puesto que no asegura su introducción al mercado.

f) Las universidades deben implementar sistemas de auditoría que les permita controlar la entrada efectiva de ingresos por las patentes licenciadas.

El objeto en estudio identificado es la idea o proyecto cuyo resultado puede dar origen a una o más patentes, objeto que pasa por un conjunto de estados cuya secuencia se representa mediante un diagrama de secuencia de estados (figura 1).

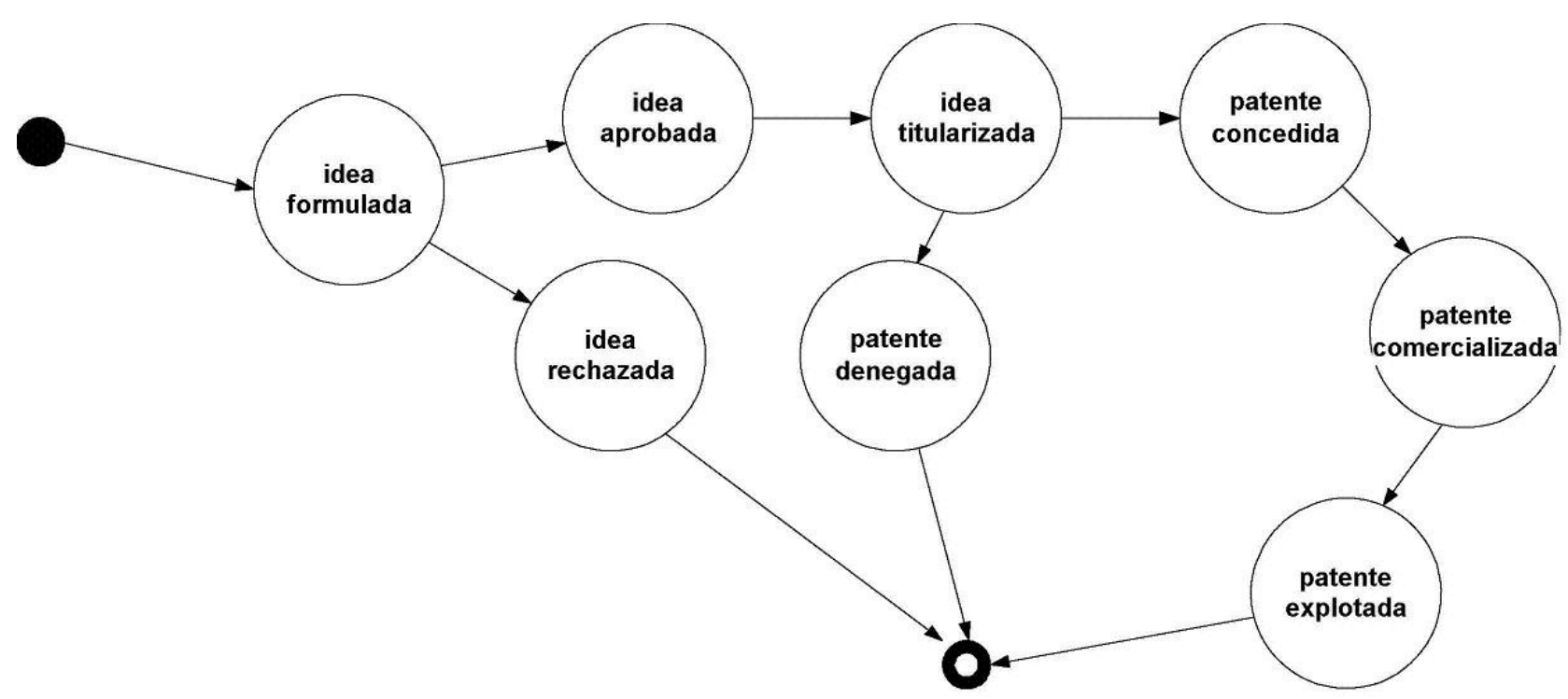

Fig.1: Diagrama de Secuencia de Estados

En la figura 2 se muestra el modelo por procesos de regulación para la Gestión de Patentes Universitarias resultante, observándose los subprocesos físicos asociados a los procesos de gestión de los derechos de propiedad intelectual en las universidades. 


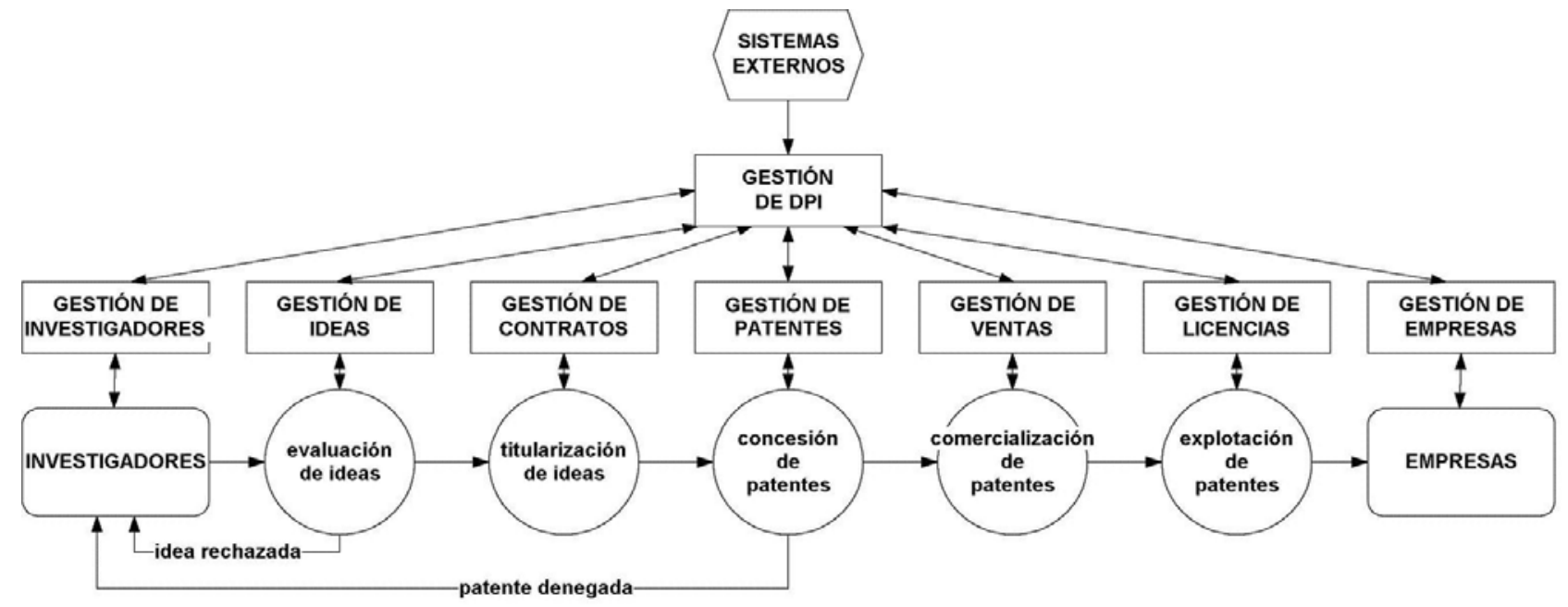

Fig.2: MPR para la Gestión de patentes en las universidades

Posteriormente se tradujo este modelo a una notación BPMN que se muestra en la figura 3.

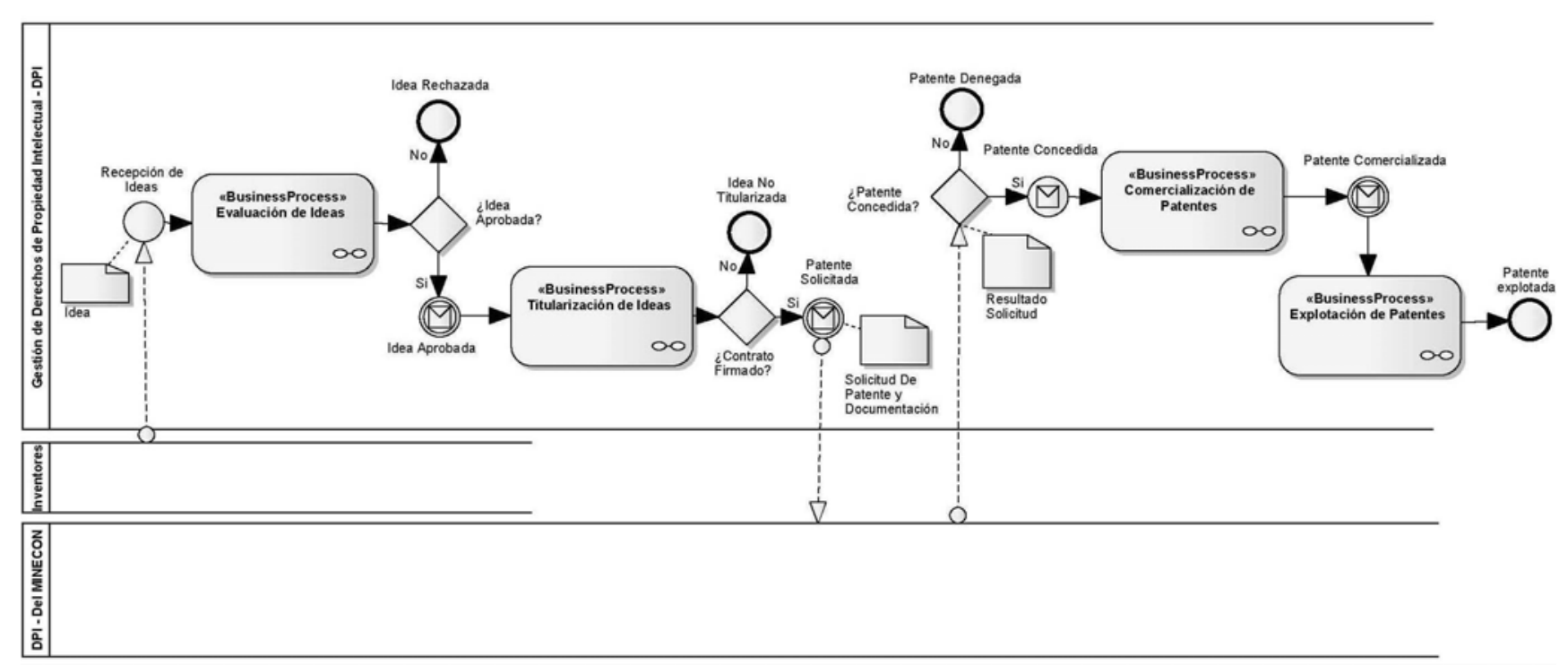

Fig.3: BPMN para la Gestión de patentes en las universidades

\section{DESCRIPCIÓN DE LOS SUBPROCESOS}

\section{Subprocesos físicos}

1. Subproceso de evaluación de ideas/proyectos y búsqueda de financiación: Su objetivo es detectar si los resultados de las ideas/ proyectos son susceptibles de protección. Para ello, los proyectos de investigación se evalúan y clasifican desde el derecho de propiedad intelectual. Si bien una primera clasificación puede ser efectuada por el investigador, ella debe estar refrendada por la unidad que gestiona los derechos de propiedad intelectual. Si se espera producir una patente derivada del proyecto, la unidad de gestión de los derechos de propiedad intelectual, procederá con el estudio de la técnica y la comprobación previa de la novedad, hasta verificar si el resultado es patentable. Como resultado de la evaluación, la idea/proyecto puede ser aprobada o rechazada. En este último caso se asume que es con expresión de causa, pudiéndose hacer sugerencias de modificación para una nueva presentación.

2. Subproceso de titularización de ideas/proyectos: Es el subproceso por el cual las ideas aprobadas se especifican en un contrato, en el cual se estipulan los compromisos que contraen las 
partes involucradas (investigadores, patrocinadores, socios, empresas, laboratorios, etc.), e incluye todas las actividades necesarias conducentes a completar la solicitud de patente correspondiente, la que es presentada ante la instancia oficial pertinente en los términos exigidos.

3. Subproceso de concesión de patentes: Esta etapa tiene como objetivo realizar un acompañamiento permanente al investigador en la obtención y mantenimiento de la patente, lo que implica total disponibilidad para la atención de las observaciones que desde la instancia oficial se formulen. Adicionalmente, se registran los alcances, las observaciones, las rectificaciones y demás que correspondan al proceso.

4. Subproceso de comercialización de patentes: Este subproceso incluye la valoración de la patente, el proceso de oferta y finalmente debiera culminar con la venta de la patente o el otorgamiento de una licencia. La formalización del proceso se hace mediante contratos de compra venta, contratos de transferencia de tecnología, contratos de licencias de explotación y contratos de know-how. Bajo el paradigma de la comercialización, con el fin de comercializar sus proyectos de creación de nuevos conocimientos y sus productos tecnológicos derivados, las universidades están creando estructuras de interfaz tales como Oficinas de Transferencia Tecnológica (OTTs), Oficinas de Transferencia de Resultados de Investigación (OTRIs) y spin off, entre otros.

5. Subproceso de explotación de patentes: Es el subproceso por el cual las empresas explotan las patentes, las que pueden haber sido compradas o licenciadas y la universidad recibe los ingresos por la venta de la patente o los royalties en caso de haberse licenciado.

\section{Subprocesos administrativos}

Identificadas las actividades físicas involucradas en el proceso de gestión de los derechos de propiedad intelectual en las universidades, se determinaron las actividades administrativas que las regulan. Por cada actividad física identificada deberá existir una actividad administrativa reguladora. Además, tanto el ambiente de entrada (investigadores), como el ambiente de salida (mercado), deben ser monitoreados, dando origen a sendas actividades administrativas. Los subprocesos administrativos resultantes son:

1. Subproceso de Gestión de Investigadores: Es el responsable de registrar información respecto de las capacidades, antecedentes, perfeccionamientos, proyectos y resultados de los investigadores en sus respectivas áreas disciplinarias. Incluye la actualización de la información disponible y la elaboración de estadísticas.

2. Subproceso de Gestión de Ideas/Proyectos: Es el responsable de registrar y monitorear los resultados de las evaluaciones de las ideas/proyectos formulados junto a un portafolio de problemas no resueltos y de potenciales empresas patrocinantes o socias, y la disponibilidad de bases de datos que sirvan de apoyo a los responsables de las evaluaciones.

3. Subproceso de Gestión de Contratos o Formalización de los Proyectos: Es el responsable de generar los contratos con los investigadores, empresas patrocinantes o socias involucrados conteniendo los compromisos de las partes, las actividades a llevarse a cabo, conducentes a las solicitudes de patentes junto con controlar el cumplimiento de las exigencias legales correspondientes.

4. Subproceso de Gestión de Patentes: Es el responsable de efectuar un seguimiento de las solicitudes de patentes con miras a que sean concedidas, registrando los alcances, observaciones, rectificaciones, etc. que correspondan.

5. Subproceso de Gestión de Ventas: Es el responsable de regular la venta de las patentes o de la entrega de licencias para su explotación por parte de terceros. Este proceso se activa cada vez que se vende una patente o se otorga una licencia a un tercero que lo autoriza a producir el objeto patentado. 
6. Subproceso de Gestión de Licencias: Es el responsable de gestionar los ingresos que reporte la explotación de las licencias otorgadas por las patentes. Este subproceso es activado cuando las empresas se encuentran produciendo el objeto patentado en virtud de la licencia que les ha sido otorgada.

7. Subproceso de Gestión de Empresas: Es el responsable de registrar información respecto de las empresas con las cuales interesa vincularse para los efectos de comercializar y explotar las patentes y licencias. Incluye la actualización de la información disponible y la elaboración de estadísticas.

Es importante destacar que las fronteras definidas para cada uno de los procesos, en la práctica son más borrosas que nítidas, pudiendo solaparse entre ellos. A modo de ejemplo en el subproceso de gestión de ideas pueden darse actividades que sean también del subproceso de gestión de ventas, lo que suele presentarse cuando la negociación se lleva a cabo en la fase de desarrollo del concepto/idea.

\section{CONCLUSIONES}

El modelo elaborado, basado en procesos claramente delimitados, aspira contribuir a la creación de una institucionalidad dentro de las universidades en un ámbito de poco desarrollo. Para aquellas universidades, interesadas en que los resultados de sus proyectos de investigación no se limiten a publicaciones indexadas, es de particular importancia disponer de una estructura organizacional que apoye y facilite la producción de patentes por parte de sus investigadores.

El resultado obtenido fue un modelo conformado por un conjunto de procesos que se pueden asociar a unidades organizacionales, las cuales pueden ser internas, esto es, encontrarse dentro de la universidad, como externas, si es que se opta por la externalización o tercerización(outsourcing), lo que dependerá de la relevancia, estrategia y/o recursos internos disponibles en cada universidad.

Por razones de simplificación, el modelo elaborado supone una cierta linealidad o secuencialidad en los procesos, asumiendo en un extremo a los investigadores de las universidades como los generadores de ideas, proyectos cuyos resultados pueden dar origen a patentes, pero cuyas ideas y proyectos no necesariamente apunten a resolver problemas de las empresas; y en el otro extremo, las empresas encargadas de explotar las patentes. Esta desvinculación si bien puede ser válida bajo una manera de ver la ciencia y la tecnología, la tendencia apunta hacia un mayor relacionamiento universidad-empresa de modo que las ideas/proyectos que emerjan de los investigadores estén destinados a resolver problemas reales no resueltos a la fecha, y que por tanto puedan dar origen a patentes. En este plano una próxima tarea consistirá en transformar el modelo en un modelo circular que considere esta necesaria asociación entre investigadores y empresas.

En consecuencia, se concluye que el modelo construido está destinado a facilitar el proceso de gestión de patentes en las universidades particularmente interesadas en su promoción y en un mayor relacionamiento entre sus investigadores y las empresas con las cuales aspira interactuar, así como posibilitar un apropiado seguimiento de responsabilidades y tiempos dentro de la cadena que va desde que surge una idea o proyecto hasta que ella se concreta en una patente.

\section{REFERENCIAS}

Aguilar-Savén, R.S.; Business process modelling: Review and framework, Int. J. Production Economics: 90(2), 129-149 (2004).

Argyres, N.S. y Liebeskind, J.P.; Privatizing the intellectual commons: universities and the commercialization of biotechnology, Journal of Economic Behavior and Organization: 35, 427-454 (1998).

Azagra, J., Izquierdo, L.; Jiménez, F. y Serra, P.; Factores determinantes de la generación de patentes universitarias: el caso de la Universidad Politécnica de Valencia, Documento ES.3.226. Instituto de Gestión de la Innovación y el Conocimiento (INGENIO), Valencia (2001). 
Azagra, J.; La contribución de las universidades a la innovación. Tesis doctoral. Universidad de Valencia (2004).

Azagra, J. y Romero, A.; Los determinantes institucionales de las patentes del Consejo Superior de Investigaciones Científicas: una aproximación histórica y una dialéctica con la Economía, Revista Española de Documentación Científica: 32(2), 9-33 (2009).

Baldini, N., Grimaldi, R. y Sobrero, M.; Institucional changes and the commercialization of academia knowledge: a study of italian universities patenting activities between 1965 and 2002, CRESCO, Working Paper 11 (2004).

Baldini, N.; Patenting in Universities. University patenting and licensing activity: a review of the literature, Research evaluation: 15(3), 197-207 (2006).

Barros, O.; Reingeniería de Procesos de Negocios: un enfoque metodológico, $2^{\mathrm{a}}$ edición, Editorial DOLMEN, Santiago, Chile (1995).

Bessen, J. y Hunt, R.; The software patent experiment. 2004. Disponible en: http://www.researchoninnovation.org/softpat.pdf, Acceso: 20 diciembre 2009

Branscomb, L, Kodama F. y Florida R.; Industrializing Knowledge. Cambridge, MA MIT Press (1999).

Calderini, M., Franzoni, C. y Vezzulli, A.; The Unequal Benefits of Academic Patenting for Science and Engineering Research, IEEE Transactions on Engineering Management: 56(1), 16-30 (2009).

Carlsson, B., Jacobsson, S., Holmen, M. y Rickne, A.; Innovation Systems: analytical and methodological issues, Research Policy: 31(2), 233-245 (2002).

Chang, Y., Chen, M., Hua, M. y Yang P.; Managing academic innovation in Taiwan: Towardas a "scientific-economic" framework, Technological Forecasting and Social Change: 73, 199-213 (2006).

Cohen, W. Nelson, R. y Walsh, J.; Links and impacts: the influence of public research on industrial R\&D, Management Science: 48(1), 1-23 (2002).

Cohen, W. y Walsh, J.; Public research, patents and implications for industrial R\& D in the drugs, biotechnology, semiconductor and computer industries en C. W. Wessner (Ed.): Capitalizing on New Needs and New Opportunities: Government-Industry Partnerships in Biotechnology and Information Technologies. National Academy of Sciences Press. Washington, DC; 223-243 (2001). Disponible en http://www.nap.edu/catalog/10281.html

Coupe, T.; Science is Golden: Academic R\&D and University Patents, Journal of Technology Transfer: 28(1), 31-46 (2003).

Curtis, B. Kellner, M.I. y Over J.; Process modeling, Communications ACM: 35(9), 75-90 (1992).

Davenport, T.H.; Process Innovation: Reengineering Work through Information Technology. Harvard Business Scholll Press, Boston, MA, USA (1993).

Fernández de Lucio, I. y Represa D. (1998); La política y la Gestión de la Propiedad Industrial en un Centro Público de Investigación. Curs de Gestió De Projectes d'I+D i Innovació en Cooperació. IMEDEA. Disponible en

http://www.imedea.uib.es/public/cursoid/html/textos/Tema\%2010.4\%20doc\%20compl.pdf, Acceso: 15 enero de 2010

Foltz, J., Barham, B. y Kim K.; Universities and Agricultural Biotechnology Patent Production, Working Paper (2000). 
Geuna, A. y Nesta, L.; University patenting and its effects on academic research: The emerging European evidence, Research Policy: 35, 795 - 807 (2006).

Gulbrandsen, M.; Rapmund, A. y Iversen, E.; Academics as patent inventors. Experiences with academic patenting when researchers own the intellectual property rights". Paper para 5th Triple Helix Conference, 18-21 de mayo, Turin (2005).

Hammer, M., y Champy, J.; Re-engineering the Corporation: A Manifesto for Business Revolution. Harper Business, New York, USA (1993).

Henderson, R., Jaffe, A. y Trajtenberg, M.; Universities as a source of commercial technology: A detailed analysis of university patenting, 1965-1988, The Review of Economics and Statistics: 80, 119-127 (1998).

Iversen, E., Gulbrandsen, M. y Klitkou, A.; A baseline for the impact of academic patenting legislation in Norway." Scientometrics: 70(2), 393-414 (2007).

Jensen, R. y Thursby, M.; Proofs and Prototypes for Sale: The Licensing of University Inventions. The American Economic Review: 91(1), 240 -259 (2001).

Kline, S. y Rosenberg, N.; An Overview of Innovation en: Landau/ Rosenberg (Ed.) The positive Sum Strategy, Harnessing Technology for Economic Growth, National Academy Press, Washington, D.C., 275-305 (1986).

Kosanke, K.; Business Process Modelling and Standarisation (2003). Disponible en http://www.cimosa.de/Stnadards/BPM_and_Standarisation.pdf, Acceso: 10 enero de 2010.

Levitt, T.; Marketing Myopia. Harvard Business Review: 38(4), 45-56 (1960).

López, M.S., Schmal, R., Cabrales, F., Mejía, J., Hidalgo, L. y García, C.; Gestión del Conocimiento con Aplicaciones Comerciales en Universidades: Patentes y Licencias". Informe Proyecto de Investigación financiado por el Comité de Desarrollo de la Investigación - CODI de la U. de Antioquia. Registro: E0077, patrocinado por CONICYT - Chile y COLCIENCIAS - Colombia, 1 - 265 (2006).

López, M.S.; Hacia una gestión universitaria de los derechos de propiedad industrial: patentes. Tesis Doctoral. Universidad del País Vasco/Euskal Herriko Unibertsitatea, España (2008).

Lopez, M.S., Schmal, R., Cabrales, F. y García C.; Los procesos en un modelo de gestión de patentes universitarias, Revista Ingeniería e Investigación de la Universidad Nacional de Colombia, Bogotá: 29(2), 135-141 (2009).

Macho-Stadler, I., Perez-Castillo, D. y Vengelus, R; Licensing of university inventions: the role of a technology transfer office. International Journal of Industrial Organization: 25, 483-510 (2007).

McAdam, R., Keogh, W., Galbraith, B. Y Laurie, D.; Defining and improving technology transfer business and management processes in university innovation centres. Tecnhnovation: 25, 1418-29 (2005).

Mowery, D., Nelson, R., Sampat, B y Ziedonis, A.; The growth of patenting and licensing by U.S universities: an assessment of the effects of the Bayh Dole act of 1980, Research Policy: 30, 99-119 (2001).

Mowery, D. y Sampat, B.; University patents and patent policy debates in the USA, 1925-1980. Industrial and Corporate Change, 10, 781-814 (2001). 
Mowery, D., Nelson, R, Sampat, B. y Ziedonis A.; Ivory Tower and Industrial Innovation. Stanford, CA: Stanford University Press (2004).

Nelson, R.; The market economy and the scientific commons, Research Policy, 33(3), 455-471 (2004).

OCDE; Turning Science into Business. Patenting and licensing at public research organizations. OCDE, París (2003).

Payne, A. y Siow, A.; Does federal Research Funding Increase University Research Output?, Advances in Economic Analysis \& Policy: 3(1), 1-24 (2003).

Rasmussen, E., Moen, O. y Gulbrandsen, M; Iniciatives to promote commercialization of university knowledge, Technovation: 26, 518-33 (2006)

Sampat, B., Mowery, D. y Ziedonis; A. Changes in university patent quality after the Bayh-Dole Act: A re-examination, International Journal of Industrial Organization: 21, 1371-1390 (2003).

Siegel, D., Waldman, D. y Link, A.; Assessing the impact of organizational practices on the productivity of university technology transfer offices: An exploratory study, Research Policy: 32, 27-48 (2003).

Siegel, D., Waldman, D., Leanne, A. y Link, A.; Toward a model of the effective transfer of scientific knowledge from academicians to practitioners: qualitative evidence from the commercialization of university technologies. Journal of Engineering and Technology Management: 21(1-2), 115-142 (2004).

Tijssen, R.; Is the commercialisation of scientific research affecting the production of public knowledge? Global trends in the output of corporate research articles, Research Policy: 32, 709-733 (2004).

Tornatzky, L.G., Waugaman, P.G. y Gray, D.O.; Innovation University: New University Roles In A Knowledge Economy, Southern Technology Council and Southern Growth Policies Board, Research Triangle Park, NC, USA (2002).

Thursby, J., Fuller, A.W. y Thursby M.; US faculty patenting: Inside and outside university. Research Policy: 38, 14-25 (2009).

White, S.A. y Miers D.; BPMN Modeling and reference guide, Future Strategies Inc. Home, USA (2008). 
